\title{
Unsupervised segmentation of MRI knees using Image Partition Forests
}

\author{
Marija Marčan and Irina Voiculescu \\ University of Oxford, Department of Computer Science
}

\begin{abstract}
Nowadays many people are affected by arthritis, a condition of the joints with limited prevention measures, but with various options of treatment the most radical of which is surgical. In order for surgery to be successful, it relies on careful analysis of patient-based models generated from medical images, usually by manual segmentation. In this work we show how to automate the segmentation of a crucial and complex joint - the knee. To achieve this goal we rely on our novel way of representing a $3 \mathrm{D}$ voxel volume as a hierarchical structure of partitions which we have named Image Partition Forest (IPF). The IPF contains several partition layers of increasing coarseness, with partitions nested across layers in the form of adjacency graphs. On the basis of a set of properties (size, mean intensity, coordinates) of each node in the IPF we classify nodes into different features. Values indicating whether or not any particular node belongs to the femur or tibia are assigned through node filtering and nodebased region growing. So far we have evaluated our method on 15 MRI knee scans. Our unsupervised segmentation compared against a hand-segmented gold standard has achieved an average Dice similarity coefficient of 0.87 for both femur and tibia, and an average symmetric surface distance of $3.77 \mathrm{~mm}$ for femur and $1.52 \mathrm{~mm}$ for tibia. The paper also discusses ways to introduce stricter morphological and spatial conditioning in the bone labelling process.
\end{abstract}

\section{Introduction}

Arthritis affects more than 10 million people in the UK of all ages, but is most common in people over 50. Why the disease occurs is not fully understood, but we know that it causes pain and inflammation as a result of both cartilage damage, and of the eventual bone-on-bone articulation when the cartilage is completely worn away [1]. From the shapes of bones, cartilage and ligaments, it is possible to predict the behaviour of an arthritic joint and assess different treatments using finite element analysis techniques.

In a clinical research context the shapes of the anatomical structures are found from single slices of CT or MRI through manual segmentation using software (such as Mimics ${ }^{\mathrm{TM}}$ or Simpleware ${ }^{\mathrm{TM}}$ ). This involves thresholding and inspection and correcting of regions which have not been detected properly. On average, the processing of a whole joint will take 4-8 user hours. In order to relieve the engineers and clinicians of the cumbersome process of manual segmentation we have directed our work towards developing methods for automatic segmentation of joint structures. The current focus of our work is the joint which is most affected by osteoarthritis - the knee, and the most appropriate imaging modality for it - MRI.

Recent literature reports several attempts in automatic segmentation of the knee structures (mainly femur, tibia and the cartilage) with various success [2]. Most successful methods so far were based on active appearance [3]. Irregardless of the specifics, common characteristic of all methods is in them processing the MRI images as an array of pixels. In our method we utilise a different approach to image segmentation which partitions the image into similar regions and then groups the partitions on several levels to create a specific data structure named image partition forest (IPF) [4]. Such structure is then analysed in order to identify relevant features (bones, organs and blood vessels). The IPF method has previously been thoroughly tested on abdominal CT scans, with good results.

In this work we present the results of the first steps towards utilising IPFs in order to automatically segment knee structures from MRI images. Here we focus on identifying femur and tibia from the partitioned image as the most prominent features in the knee and a starting point for identifying other structures such as cartilage and meniscii.

\section{Materials and Methods}

\section{Image Partition Forest}

As described in our previous work [4, 5], an image partition forest is a data structure used to represent an image differently from the standard one which treats the image as an array of pixels. It is a tree-based representation in which each node corresponds to one region (partition) of the image while the leaf nodes (i.e. the nodes in the lowest level of the tree) are in fact pixels. The 


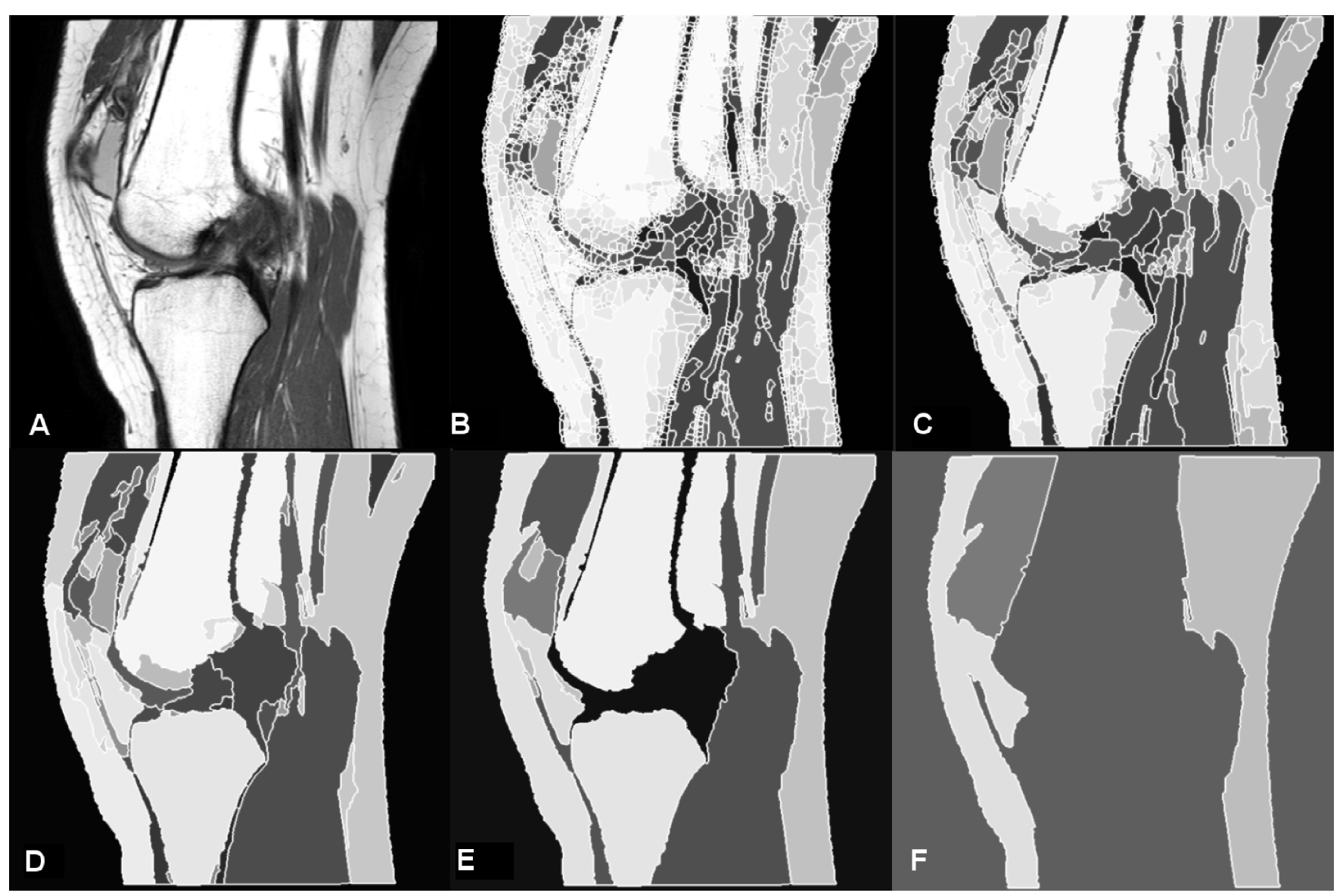

Figure 1: Different layers of an IPF. A. Original MRI image of the knee. B. - F. Layers 1 through 5, representing image partitions in decreasing detail level.

image partition forest consists of several levels of nodes with each higher level containing less regions than the previous one. The union of all nodes in one level corresponds to the whole image. All nodes in the forest are organized in adjacency graphs which enables us to easily find and identify adjacent regions as well as subregions. An example of an IPF of an MRI image of the knee can be seen in Figure 1 along with the original MRI image.

Construction of the IPF is performed by a bottom-up approach. The image is first run through a watershed algorithm which creates the lowest-level partitions. The higher level partitions are then created by subsequent runs of the waterfall algorithm which naturally groups similar and adjacent partitions into larger ones. The process of IPF construction has been implemented so that it can partition the image both in $3 \mathrm{D}$ and also on the $2 \mathrm{D}$ level of individual slices. After the forest structure has been formed each node is assigned values of certain properties. The properties that are currently calculated are mean intensity of the region area, size of the area, minimum and maximum coordinates and minimum and maximum intensity values.

\section{Feature identifier for femur and tibia}

The IPF representation is useful for the feature identification step, in that it provides a helpful space in which to search for image features of different kinds and sizes. In order to identify the femur and tibia from the IPF we perform node filtering which takes into account several region properties and criteria. The criteria set has been constructed based on visual analysis of the image partition forest of the knee images. Both femur and tibia appear as large relatively bright areas in higher levels of the IPF. However, due to inhomogeneity of the bone appearance near the knee joint that area might contain several smaller partitions even in the higher levels. Also the edge of the bones near the knee joint may not always be well delineated in large partitions. The approach of feature identification is therefore performed in two steps. In the first step we identify the largest regions in the middle part of the image as candidates for femur and tibia. In the second step we try to identify the exact border of the structures by performing region growing on the lowest partition level with children of the regions from previous step as seeds. The criteria for the region growing takes into account the intensity similarity between adjacent nodes and also incorporates spatial criteria formed based on the bounding box of the candidate regions from step one. All of the parameters that were used in our initial 
experiments were chosen by quick visual inspection of several MRI cases from the database at hand. The parameters in question were namely the usual position of the femur and tibia in the image, their average size in one slice, their intensity variance, and the intensity difference regarding neighboring areas.

\section{Dataset}

Although there is a large number of different knee MRI sequences present today, we initially had only one type at our disposal. Since the images we obtained were already anonymized they also lacked the info about the exact sequence, but based on image appearance they could be identified as saggital, intermediate-weighted knee scans without fat suppression. The pixel spacing was $0.3 \times 0.3 \mathrm{~mm}$ and the slice distance was $2.5 \mathrm{~mm}$. We have received a total of 15 of such image volumes along with manual segmentations which were performed using Mimics $^{\mathrm{TM}}$ by a biomedical engineer working in numerical modelling of the kneeZZ.

\section{Results}

We have run our unsupervised algorithm based on IPFs on all 15 MRI images and compared them to manual segmentations. For each case we calculated the values of sensitivity, specificity, Dice similarity coefficient, average symmetric surface distance (ASSD) (as defined in [2]) and Hausdorff distance.

For femur mean value of sensitivity was 0.79 , mean value of specificity 0.99 , mean value of ASSD $3.77 \mathrm{~mm}$, and mean value of Hausdorff distance $54.3 \mathrm{~mm}$. For tibia mean value of sensitivity was 0.83 , mean value of specificity 0.99 , mean value of ASSD $1.52 \mathrm{~mm}$, and mean value of Hausdorff distance $7.63 \mathrm{~mm}$.

In Figure 2 we provide visual example of the results of the feature identification process for one of the cases from our dataset, both on slice level and as a 3D model.

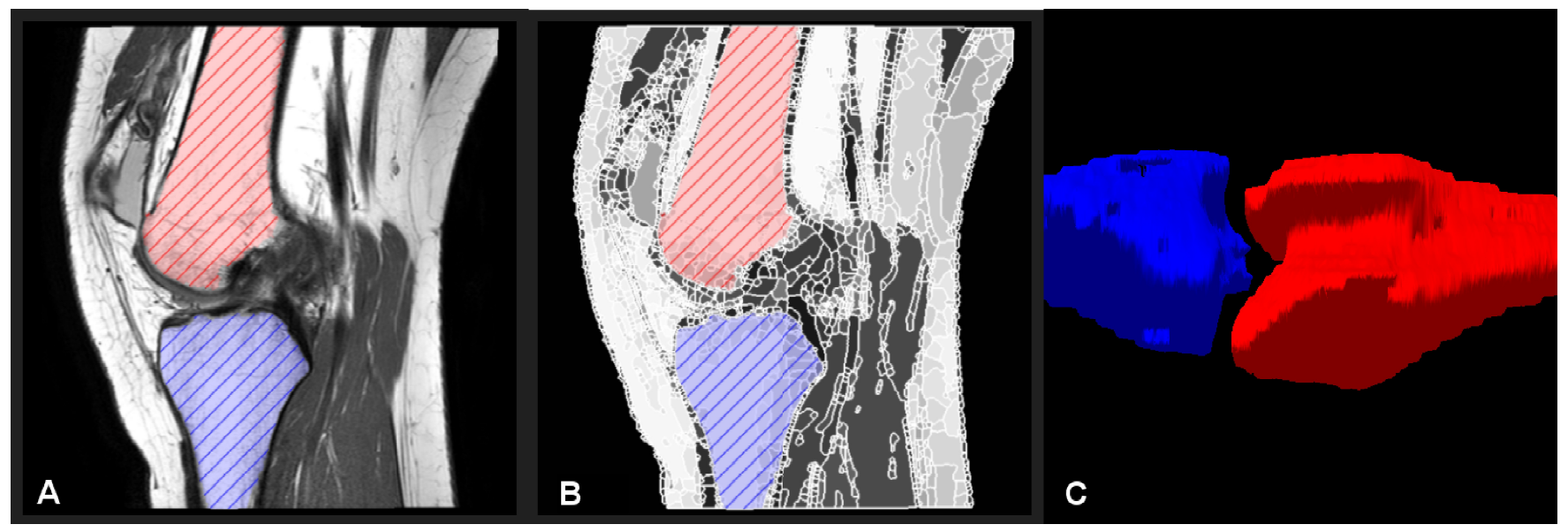

Figure 2: Results of feature identification of IPF. Red is femur, blue is tibia. A. Unpartitioned image. B. Partitioned image. C. $3 \mathrm{D}$ visualization of the result.

\section{Discussion and Future Work}

In the presented work we applied a novel approach to image representation called image partition forest on the MRI images of the knee in order to automatically identify femur and tibia.

Compared to the state of the art methods for segmentation of femur and tibia presented at the MICCAI Grand Challenge in knee segmentation in 2010 [2,3] our method performs less accurately. The best method from the named challenge achieved an average symmetric surface distance of $0.42 \mathrm{~mm}$ for femur and $0.38 \mathrm{~mm}$ for tibia, while our method scored $3.77 \mathrm{~mm}$ and $1.52 \mathrm{~mm}$ in the respective metrics. Although the dataset used for validation of our method is different from the one used in the MICCAI challenge, especially regarding the choice of the MRI sequence, it is currently the best possible way of ranking our method on the global scale. We do however plan to modify and evaluate our method on the dataset used in the MICCAI challenge as well (we were unfortunately unable to obtain it in sufficient time prior to abstract submission deadline).

Other than using IPF partitioning to segment the image, other main difference between our method and most of state-of-the-art approaches is in the fact that we do not perform any training in order to optimize method performance. All of the parameters 
that were used in our initial experiments were chosen by quick visual inspection of several MRI cases from the database at hand. Due to quite an amount of variability between different knees, not optimizing the method on a larger database might be a drawback. While this is a valid point to be explored, we believe the method would benefit more through integration of more strict spatial conditioning, possibly along the lines of active shape models.

Another possible path of exploration in order to improve our method would be by improving the IPF generation itself. The final result of feature identification is heavily reliant on the way the image is partitioned. Although our previous work also included a way of interactive manipulation with the IPF and partitions, there is currently no way to modify the results of the partitioning without human supervision. Unfortunately, the partitioning is not always perfect, in a way that a partition can sometimes contain more than one feature which subsequently causes the region growing algorithm to leak into undesired areas. This might be circumvented by different preprocessing of the image (in terms of windowing or filtering) in order to ensure correct partitioning of the desired features.

What should be noted when dealing with segmentation of knee structures from MRI is the vast choice of MRI sequences. Given the fact that there is at least a dozen of different MRI sequences reported in the literature with a different appearance of various knee structures [6], a choice of sequence is something that must be taken into account when aiming to automatically extract knee structures from MRI images. We were thus far able to obtain only one type of sequence but aim to work with others, specifically those that would enable us to segment cartilage. Such sequences, defined in the literature as sequences for quantitative measurement of cartilage [6], are usually 3D and have isotropic voxels. Using images with isotropic voxels could also be beneficial for accurate extraction of femur and tibia. As can be noted in Figure 2.C, even such large structures like femur and tibia do suffer from relatively large intra-slice distances $(2.5 \mathrm{~mm})$ which causes the staircase effect in 3D reconstructed model. Also, using sequences with isotropic voxels is likely to diminish the partial-volume effect and in such way improve the quality of image segmentation.

Acknowledgements We are grateful to Dr Elise Pegg of the Nuffield Department of Orthopaedics, Rehumatology and Muscoluskeletal Sciences, University of Oxford, for providing the MRI data used in validation. We are also grateful to Dr Mona Alinejad who performed the manual segmentation of the MRI scans.

\section{References}

[1] Arthritis Research UK. Musculoskeletal health: a public health approach. 2014. www.arthritisresearchuk.org/ policy-and-public-affairs/reports-and-resources/ /media/1C04D81E4C6048048E0246F588A0CD64.ashx

[2] T. Heimann, B. Morrison, M. Styner et al. Segmentation of knee images: a grand challenge. In Medical Image Analysis for the Clinic: A Grand Challenge. 2010. pp. 207-214.

[3] G. Vincent, C. Wolstenholme, I. Scott et al. Fully automatic segmentation of the knee joint using active appearance models. In Medical Image Analysis for the Clinic: A Grand Challenge. 2010. pp. 224-230.

[4] S. Golodetz, I. Voiculescu and S. Cameron. Automatic Spine Identification in Abdominal CT Slices using Image Partition Forests. In Proceedings of ISPA 2009. September, 2009.

[5] S. M. Golodetz, C. Nicholls, I. D. Voiculescu and S. A. Cameron. Two Tree-Based Methods for the Waterfall. In Pattern Recognition, April 2014.

[6] D. Hunter, R. Altman, F. Cicuttini et al. OARSI Clinical Trials Recommendations: Knee imaging in clinical trials in osteoarthritis. In Oesteoarthritis and Cartilage, 2015. pp. 698-715. 